\title{
Ciclo de mejora del proceso asistencial en una sala de hospitalización de cirugía general
}

\author{
A quality improvement cycle of the care process in a general surgery hospitalization ward
}

$M^{a}$ del Carmen Azorín-Samper*, José Aguilar-Jiménez, Benito Flores-Pastor y José L. Aguayo-Albasini

Servicio de Cirugía General y del Aparato Digestivo, Hospital Morales Meseguer, Murcia, España

\section{Resumen}

Antecedentes: Podrían existir carencias en el registro de datos relevantes del proceso asistencial de hospitalización quirúrgica. Objetivo: Completar un ciclo de mejora de la calidad del proceso asistencial quirúrgico en una planta de cirugía. Mediremos unos criterios de calidad estructurados, identificando las principales deficiencias, implementando medidas de mejora y reevaluando la calidad del proceso asistencial quirúrgico. Además, se valora la viabilidad de la implantación de un sistema nemotécnico de registro de actividad y de un método de evaluación nutricional. Método: Estudio comparativo $(n=60)$ antes-después del establecimiento de medidas de mejora basadas en el análisis del proceso asistencial quirúrgico. Los criterios evaluados fueron la información recibida por el paciente, el registro de la evolución clínica y el plan de cuidado establecido por el cirujano en la historia clínica, el registro de peso y talla, y la valoración nutricional. Se realizaron sesiones informativas e implementación electrónica de la nota SOAP (subjetive, objetive, assessment, plan) y de un test de valoración nutricional. Resultados: Hubo mejoría significativa en todos los criterios medidos (información, registro y evaluación nutricional). Conclusión: Algunas medidas sencillas permiten una mejoría importante en la información, la evaluación nutricional y el registro del proceso asistencial en una planta de cirugía.

Palabras Clave: Calidad asistencial. Estado nutricional. Pases de visita. Registros electrónicos de salud. Toma de decisiones clínicas.

\begin{abstract}
Background: There could be important failures in clinical data and plan records that potentially influence the surgical care process. Objective: To complete a quality of care improvement cycle on the surgical care process in the General Surgery ward rounds. Structured quality criteria were measured, in order to identify major deficiencies, to implement improvement measures and to reassess the quality of surgical care process. Furthermore, we'll value the viability the implementation of a structured registration system and nutritional assessment method. Method: Comparative quality study $(n=60)$ before-after the setting of several improvement measures derived from the analysis of the surgical care process. Evaluated criteria were the information received by the patient, adequate recording of the clinical course and plan of care established by the surgeon in the Electronic Health Record, recording of patient's weight and size and nutritional assessment. Informative sessions, subjective, objective, assessment, plan notes on the electronic clinical record and a nutritional assessment test were implemented. Results: There was a significant improvement in all measured criteria (information, data records and nutritional assessment). Conclusion: Simple organizational measures allow a significant improvement in the information process, clinical records and malnutrition risk detection in a surgical ward.
\end{abstract}

Key Words: Quality of health care. Nutritional status. Attending rounds. Electronic health records. Clinical decision-making.

Correspondencia:

*María del Carmen Azorín-Samper

Avda. Ramón y Cajal, s/n

Fecha de recepción: 28-01-2019

Cir Cir. 2020;88(2):175-184

C.P. 46520, Sagunto, Valencia, España

Fecha de aceptación: 24-07-2019

Cir Cir. 2020;88(2): 175-184

E-mail: mcazosam16@gmail.com

DOI: $10.24875 / C I R U .19001066$

www.cirugiaycirujanos.com

0009-7411/@ 2019 Academia Mexicana de Cirugía. Publicado por Permanyer. Este es un artículo open access bajo la licencia CC BY-NC-ND (http://creativecommons.org/licenses/by-nc-nd/4.0/). 


\section{Introducción}

La práctica de una asistencia de calidad es un pilar fundamental en el correcto desarrollo de la medicina clínica'. La atención a los pacientes ingresados en la planta de hospitalización de un servicio de cirugía general es un proceso complejo de toma de decisiones y gestión de cuidados que depende del conocimiento y de la experiencia de los cirujanos responsables, de la situación clínica del paciente y de la capacidad del equipo asistencial para la provisión de las medidas que se consideren adecuadas ${ }^{2}$.

El correcto registro de los datos relevantes para este proceso y del propio plan de actuación es de gran importancia para la comunicación entre los profesionales sanitarios ${ }^{3}$, en particular cuando existen distintos cirujanos (y otros profesionales) responsables de la atención, lo que en muchas ocasiones se ve dificultado por la disponibilidad de tiempo, la distribución de las cargas de trabajo en el equipo, los sistemas de registro electrónico y la falta de una sistematización adecuada en la toma de datos ${ }^{4}$.

Otros autores han evaluado el proceso de documentación y han observado importantes déficits, que llegan a incumplir los requerimientos de los organismos reguladores ${ }^{5}$. En nuestro medio se han publicado algunos trabajos sobre la calidad de los informes de alta de los servicios de cirugía $a^{6,7}$, pero hasta donde conocemos no existen publicaciones respecto al proceso de documentación de la atención quirúrgica en una planta de hospitalización.

El objetivo de nuestro estudio es completar un ciclo de mejora de calidad del proceso asistencial quirúrgico en una sala de hospitalización que incluye la incorporación de la denominada «nota SOAP» (subjetive, objetive, assessment, plan) en relación con el concepto de «historia clínica orientada por problemas" 8 , el Mini Nutritional Assesment 9 (MNA) y la mejora de la información de la que dispone el paciente sobre su proceso asistencial.

\section{Método}

\section{Diseño del estudio}

Se realizó un estudio de nivel de calidad comparativo antes-después de la implementación de unas medidas de mejora de la calidad a través del análisis del proceso asistencial en la planta de hospitalización del servicio de cirugía general del Hospital Morales Meseguer, de Murcia (España). Este servicio cuenta con 20 cirujanos y 48 camas de hospitalización. Se

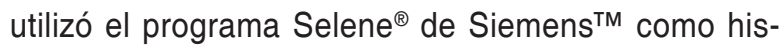
toria clínica electrónica (HCE).

\section{Tamaño muestral}

Se calculó un tamaño muestral de 53 pacientes para estimar, con una confianza del $95 \%$ y una precisión de 14 unidades porcentuales, un porcentaje poblacional que se preveía que fuese alrededor del $60 \%$ para el cumplimiento de la variable registro evolutivo correcto, la cual agrupa las variables medidas en el estudio. Se estimó una pérdida de seguimiento del $10 \%{ }^{10}$.

\section{Sujetos y períodos de estudio}

Durante el estudio se realizaron dos evaluaciones, incluyendo de forma consecutiva 60 pacientes ingresados en la planta de cirugía general y digestiva en cada período de estudio, suponiendo un total de 120 .

La evaluación inicial se realizó durante una semana de mayo de 2015 y, tras implantar en junio de 2015 las medidas de mejora, se realizó una segunda evaluación durante una semana de noviembre de 2015.

Los criterios de inclusión fueron ser pacientes ingresados en el servicio de cirugía, tanto por enfermedad quirúrgica como médica. Los criterios de exclusión fueron ser pacientes con estancia hospitalaria inferior a 1 día.

\section{Ciclo de mejora}

El cirujano adjunto responsable del proyecto y la autora principal elaboraron y definieron los criterios, sus dimensiones, tipos y fuente de datos (Tabla 1), y una hoja de evaluación de cumplimientos que fue recogida por la autora principal. Los componentes evaluados fueron la información recibida por el paciente, el registro de la evolución clínica (subjetiva y objetiva) y el plan de cuidado establecido por el cirujano en la HCE durante los 7 días previos, y la valoración nutricional. Se elaboró un diagrama de causa-efecto (Fig. 1). Tras este análisis inicial se diseñaron e implantaron diferentes medidas de mejora. Tras ello se realizó la medida de cumplimientos después de la implementación de las medidas y se hizo la comparación antes-después. 
M.C. Azorín-Samper, et al.: Ciclo de mejora del proceso asistencial en cirugía general

Tabla 1. Criterios, dimensiones, tipos y fuentes de los datos evaluados

\begin{tabular}{|c|c|c|c|c|c|c|c|}
\hline Indicador & Aclaraciones & Excepciones & $\begin{array}{l}\text { Tipo de } \\
\text { indicador }\end{array}$ & $\begin{array}{l}\text { Dimensión } \\
\text { estudiada }\end{array}$ & Justificación & $\begin{array}{l}\text { Fuente de } \\
\text { datos }\end{array}$ & $\begin{array}{l}\text { Origen del } \\
\text { indicador }\end{array}$ \\
\hline $\begin{array}{l}\text { Médico } \\
\text { responsable: } \\
\text { el paciente } \\
\text { conoce al médico } \\
\text { responsable de su } \\
\text { caso }\end{array}$ & $\begin{array}{l}\text { Se considerará } \\
\text { afirmativo siempre } \\
\text { que el paciente } \\
\text { indique el nombre o } \\
\text { apellido del cirujano } \\
\text { responsable de su } \\
\text { caso. } \\
\text { Por ejemplo: } \\
\text { "Dr. Lucas» o } \\
\text { "Dr. López» }\end{array}$ & & Proceso & $\begin{array}{l}\text { Calidad de } \\
\text { satisfacción }\end{array}$ & $\begin{array}{l}\text { La medida } \\
\text { del indicador } \\
\text { permitirá } \\
\text { valorar si el } \\
\text { paciente está } \\
\text { bien informado, } \\
\text { si sabe quién } \\
\text { es su cirujano } \\
\text { responsable } \\
\text { o quién está } \\
\text { a cargo del } \\
\text { servicio de } \\
\text { cirugía general, } \\
\text { lo que valorará } \\
\text { la calidad de } \\
\text { información } \\
\text { recibida por el } \\
\text { paciente }\end{array}$ & $\begin{array}{l}\text { Se obtendrá } \\
\text { mediante la } \\
\text { realización de } \\
\text { una encuesta } \\
\text { por el autor } \\
\text { principal del } \\
\text { estudio a los } \\
\text { pacientes } \\
\text { ingresados en la } \\
\text { sala de cirugía, } \\
\text { por motivo } \\
\text { quirúrgico } \\
\text { o médico, } \\
\text { mediante una } \\
\text { entrevista }\end{array}$ & $\begin{array}{l}\text { Elaboración } \\
\text { propia a } \\
\text { través del } \\
\text { cuestionario } \\
\text { formulado }\end{array}$ \\
\hline $\begin{array}{l}\text { Procedimiento: } \\
\text { el paciente conoce } \\
\text { el procedimiento o } \\
\text { el propósito de la } \\
\text { intervención }\end{array}$ & $\begin{array}{l}\text { Se considerará } \\
\text { afirmativo si el } \\
\text { paciente conoce el } \\
\text { motivo de su ingreso } \\
\text { o el objetivo sin ser } \\
\text { condición necesaria } \\
\text { que lo indique con } \\
\text { palabras técnicas. } \\
\text { Por ejemplo: } \\
\text { «Me han quitado } \\
\text { la vesícula porque } \\
\text { tenía piedras» }\end{array}$ & & Proceso & $\begin{array}{l}\text { Calidad de } \\
\text { satisfacción }\end{array}$ & $\begin{array}{l}\text { La medida } \\
\text { del indicador } \\
\text { permitirá medir si } \\
\text { el paciente está } \\
\text { bien informado } \\
\text { del motivo de } \\
\text { su ingreso, } \\
\text { se evaluará } \\
\text { la calidad de } \\
\text { información } \\
\text { proporcionada } \\
\text { al paciente por } \\
\text { parte del } \\
\text { personal médico }\end{array}$ & $\begin{array}{l}\text { Se obtendrá } \\
\text { mediante la } \\
\text { realización de } \\
\text { una encuesta } \\
\text { por el autor } \\
\text { principal del } \\
\text { estudio a los } \\
\text { pacientes } \\
\text { ingresados en la } \\
\text { sala de cirugía, } \\
\text { por motivo } \\
\text { quirúrgico } \\
\text { o médico, } \\
\text { mediante una } \\
\text { entrevista }\end{array}$ & $\begin{array}{l}\text { Elaboración } \\
\text { propia a } \\
\text { través del } \\
\text { cuestionario } \\
\text { formulado }\end{array}$ \\
\hline $\begin{array}{l}\text { Registro de } \\
\text { actividad: } \\
\text { descripción del } \\
\text { proceso }\end{array}$ & $\begin{array}{l}\text { Se considerará } \\
\text { afirmativa cualquier } \\
\text { narrativa descriptiva } \\
\text { de la situación } \\
\text { actual, en la que } \\
\text { debe explicitar } \\
\text { el diagnóstico } \\
\text { en todos los } \\
\text { días revisados, } \\
\text { tomando un período } \\
\text { de } 7 \text { días. Por } \\
\text { ejemplo: «2 } 2^{\circ} \text { día } \\
\text { posoperatorio de } \\
\text { apendicitis aguda» }\end{array}$ & $\begin{array}{l}\text { No se } \\
\text { considerará } \\
\text { válido el } \\
\text { indicador si se } \\
\text { hallan, aunque } \\
\text { sea un solo } \\
\text { día de los } \\
7 \text { revisados, } \\
\text { descripciones } \\
\text { como «Primer día } \\
\text { posoperatorio» } \\
\text { « « días de } \\
\text { ingreso» si } \\
\text { no explicita el } \\
\text { diagnóstico }\end{array}$ & Proceso & $\begin{array}{l}\text { Calidad } \\
\text { científico-técnica }\end{array}$ & $\begin{array}{l}\text { La medida } \\
\text { del indicador } \\
\text { permitirá valorar } \\
\text { la calidad } \\
\text { asistencial }\end{array}$ & & \\
\hline $\begin{array}{l}\text { Registro de } \\
\text { actividad: estado } \\
\text { subjetivo del } \\
\text { paciente }\end{array}$ & $\begin{array}{l}\text { Se considerará } \\
\text { afirmativa cualquier } \\
\text { narrativa que } \\
\text { indique el estado } \\
\text { subjetivo del } \\
\text { paciente. } \\
\text { Por ejemplo: } \\
\text { «Dolor, náuseas, } \\
\text { otros síntomas», } \\
\text { "asintomático», } \\
\text { «buen estado } \\
\text { general» }\end{array}$ & $\begin{array}{l}\text { No se } \\
\text { considerará } \\
\text { válido el } \\
\text { indicador si no se } \\
\text { halla, aunque sea } \\
\text { un solo día de los } \\
7 \text { revisados, la } \\
\text { descripción del } \\
\text { estado subjetivo } \\
\text { del paciente }\end{array}$ & Proceso & $\begin{array}{l}\text { Calidad } \\
\text { científico-técnica }\end{array}$ & $\begin{array}{l}\text { La medida } \\
\text { del indicador } \\
\text { permitirá valorar } \\
\text { la calidad } \\
\text { asistencial }\end{array}$ & & \\
\hline
\end{tabular}


Cirugía y Cirujanos. 2020;88(2)

Tabla 1. Criterios, dimensiones, tipos y fuentes de los datos evaluados (Continuación)

\begin{tabular}{|c|c|c|c|c|c|c|c|}
\hline Indicador & Aclaraciones & Excepciones & $\begin{array}{l}\text { Tipo de } \\
\text { indicador }\end{array}$ & $\begin{array}{l}\text { Dimensión } \\
\text { estudiada }\end{array}$ & Justificación & $\begin{array}{l}\text { Fuente de } \\
\text { datos }\end{array}$ & $\begin{array}{l}\text { Origen del } \\
\text { indicador }\end{array}$ \\
\hline $\begin{array}{l}\text { Registro de } \\
\text { actividad: estado } \\
\text { objetivo del paciente }\end{array}$ & $\begin{array}{l}\text { Se considerará } \\
\text { afirmativa cualquier } \\
\text { narrativa incluyendo } \\
\text { necesariamente } \\
\text { el registro de } \\
\text { las constantes } \\
\text { vitales (temperatura, } \\
\text { presión arterial, } \\
\text { frecuencia cardiaca) } \\
\text { y de la herida o sitio } \\
\text { quirúrgico. } \\
\text { Por ejemplo: } \\
\text { «Afebril, constantes } \\
\text { en rango, herida con } \\
\text { buen estado, sin } \\
\text { signos inflamatorios, } \\
\text { drenaje } \\
\text { improductivo» }\end{array}$ & $\begin{array}{l}\text { No se } \\
\text { considerará } \\
\text { válido el } \\
\text { indicador si no se } \\
\text { halla, aunque sea } \\
\text { un solo día de los } \\
7 \text { revisados, la } \\
\text { descripción del } \\
\text { estado objetivo } \\
\text { del paciente }\end{array}$ & Proceso & $\begin{array}{l}\text { Calidad } \\
\text { científico-técnica }\end{array}$ & $\begin{array}{l}\text { La medida } \\
\text { del indicador } \\
\text { permitirá valorar } \\
\text { la calidad } \\
\text { asistencial }\end{array}$ & & \\
\hline $\begin{array}{l}\text { Registro de } \\
\text { actividad: } \\
\text { plan de actuación }\end{array}$ & $\begin{array}{l}\text { Se considerará } \\
\text { afirmativo si está } \\
\text { descrito un plan } \\
\text { de actuación o } \\
\text { una motivación del } \\
\text { ingreso. } \\
\text { Por ejemplo: } \\
\text { «Dieta astringente, } \\
\text { se mantiene } \\
\text { tratamiento } \\
\text { antibiótico, se retiran } \\
\text { drenajes.» }\end{array}$ & & Proceso & $\begin{array}{l}\text { Calidad } \\
\text { científico-técnica }\end{array}$ & $\begin{array}{l}\text { La medida } \\
\text { del indicador } \\
\text { permitirá valorar } \\
\text { la calidad } \\
\text { asistencial }\end{array}$ & & \\
\hline $\begin{array}{l}\text { Registro de peso } \\
\text { y talla }\end{array}$ & $\begin{array}{l}\text { Se considerará } \\
\text { afirmativo si } \\
\text { se encuentran } \\
\text { descritos el peso y } \\
\text { la talla del paciente } \\
\text { en el programa } \\
\text { informático Selene. } \\
\text { Por ejemplo: «Peso: } \\
70 \text { kg; talla: } 170 \mathrm{~cm}\end{array}$ & & Proceso & $\begin{array}{l}\text { Calidad } \\
\text { científico-técnica }\end{array}$ & $\begin{array}{l}\text { La medida } \\
\text { del indicador } \\
\text { permitirá valorar } \\
\text { si el registro } \\
\text { de datos del } \\
\text { paciente es } \\
\text { correcto y de } \\
\text { esta forma se } \\
\text { podrán calcular } \\
\text { fluidoterapia y } \\
\text { antibioticoterapia. } \\
\text { Es una medida } \\
\text { de calidad } \\
\text { asistencial }\end{array}$ & & \\
\hline $\begin{array}{l}\text { Valoración } \\
\text { nutricional con } \\
\text { Mini Nutritional } \\
\text { Assessment (MNA) }\end{array}$ & $\begin{array}{l}\text { Se utilizará el } \\
\text { MNA, en el que } \\
\text { se obtendrá } \\
\text { valoración del } \\
\text { estado nutricional } \\
\text { del paciente: } \\
\text { 12-14 puntos } \\
\text { (estado nutricional } \\
\text { normal), 8-11 } \\
\text { puntos (riesgo } \\
\text { de malnutrición), } \\
\text { O puntos } \\
\text { (malnutrición) }\end{array}$ & $\begin{array}{l}\text { No se } \\
\text { considerará } \\
\text { válido el } \\
\text { indicador si no es } \\
\text { posible obtener } \\
\text { la información } \\
\text { a través del } \\
\text { paciente o de sus } \\
\text { familiares }\end{array}$ & Proceso & $\begin{array}{l}\text { Calidad } \\
\text { científico-técnica }\end{array}$ & $\begin{array}{l}\text { La medida } \\
\text { del indicador } \\
\text { permitirá valorar } \\
\text { el estado } \\
\text { nutricional del } \\
\text { paciente. Es } \\
\text { una medida } \\
\text { de la calidad } \\
\text { asistencial }\end{array}$ & $\begin{array}{l}\text { Se obtendrá } \\
\text { mediante } \\
\text { cribado con el } \\
\text { MNA de todos } \\
\text { los pacientes } \\
\text { ingresados } \\
\text { en la planta } \\
\text { de cirugía } \\
\text { por motivo } \\
\text { quirúrgico o } \\
\text { médico }\end{array}$ & $\begin{array}{l}\text { Obtenido } \\
\text { del } \\
\text { cuestionario } \\
\text { de } \\
\text { valoración } \\
\text { nutricional } \\
\text { - Nestle } \\
\text { Nutrition }\end{array}$ \\
\hline
\end{tabular}




Médico
- Déficit de comunicación con el paciente
- Rotación personal (rotura de continuidad
asistencial)
- Mismo personal: actividad quirúrgica y
asistencial en sala de hospitalización
- Considerar irrelevantes algunos datos
- Ausencia de un sistema de registro de la
actividad específico a cumplimentar
- Ausencia de un sistema de registro en la historia
clínica electrónica de SELENE (descripción
proceso, estado objetivo y subjetivo del paciente
plan de actuación, peso y talla)
- Rotación personal
- Delimitación de identificación de problemas
Problemas de toma de decisiones en el plan
de actuación
Sistema de registro

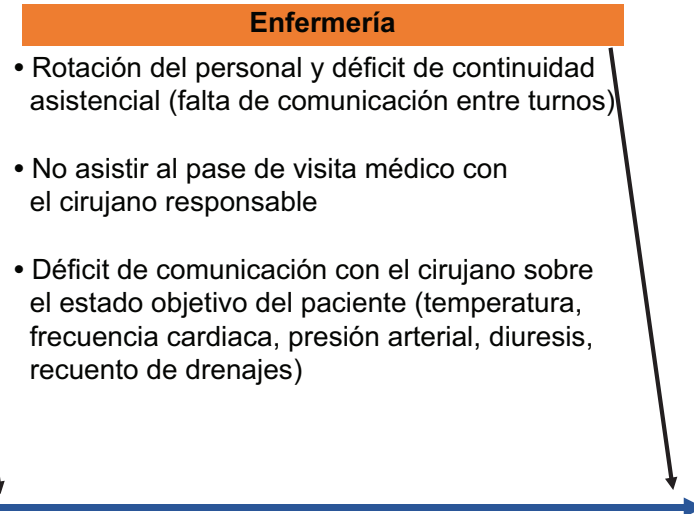

- Ausencia de una sistemática de registro de peso y talla en ingreso no programados

- Dificultad de registro de peso y talla en pacientes encamados

- Ausencia en enfermería de la aplicación de un cuestionario de valoración nutricional del paciente

Figura 1. Diagrama de Ishikawa. Análisis de causas y subcausas de la variabilidad del registro del proceso asistencial.

\section{Valoración nutricional}

Se empleó el test MNA, que recoge peso, altura y una valoración sobre seis apartados: 1) pérdida de apetito en los 3 meses anteriores; 2) pérdida de peso en los 3 meses previos; 3) movilidad; 4) enfermedad aguda 0 estrés en los 3 meses previos; 5 ) problemas de demencia o depresión; y 6) índice de masa corporal. El MNA clasifica a los pacientes en bien nutridos, con riesgo de malnutrición o con malnutrición establecida.

\section{Análisis de causas y propuesta de medidas de mejora}

Se elaboró un diagrama de causa-efecto (Fig. 1). Tras el análisis se reunió el grupo promotor del ciclo de mejora, que definió las siguientes medidas correctoras:

- Sesión informativa general a los cirujanos del servicio, en la cual se remarcaron los principales aspectos susceptibles de mejora detectados (importancia del médico responsable de una comunicación efectiva y de un registro adecuado) y las medidas propuestas.

- Implantación de la nota SOAP como sistema de registro evolutivo en la HCE durante el pase de visita médico de hospitalización realizado por dos cirujanos adjuntos y un residente de cirugía (y logotipo SOAP en los ordenadores utilizados para la visita médica en planta).

- Realización del MNA todos los martes (con carteles informativos y entrenamiento de enfermería).

- Registro de peso y talla al ingreso, y del peso semanalmente, en la HCE (con carteles informativos en la sala de cirugía y registrados por el personal de enfermería).

\section{Análisis estadístico}

Se empleó el programa estadístico SPSS, versión 20. Se realizaron el estudio descriptivo de cada serie y las comparaciones (Xi2 para tablas de contingencia) cuando fue necesario. Se hizo una comparación gráfica antes-después mediante la construcción de diagramas de Pareto. Se consideró un resultado estadísticamente significativo con un valor de $p<0.05$.

\section{Resultados}

\section{Medida inicial}

En la tabla 2 se muestra la comparación de los resultados (cumplimientos) antes y después de la intervención. Además, se destaca que en el $61.7 \%$ de los pacientes hospitalizados en la sala de cirugía no constaba en los registros evolutivos la descripción del 
Cirugía y Cirujanos. 2020;88(2)

Tabla 2. Comparación del cumplimiento de criterios antes y después de la implantación de medidas de mejora

\begin{tabular}{|c|c|c|c|}
\hline Criterios & $\begin{array}{c}\text { Cumplimiento antes } \\
\% \text { (IC 95\%) }\end{array}$ & $\begin{array}{c}\text { Cumplimiento después } \\
\% \text { (IC 95\%) }\end{array}$ & p \\
\hline 1. Médico responsable: el paciente conoce al médico responsable de su caso & $60(47.6-72.4)$ & $73.3(62.1-84.5)$ & 0.07 \\
\hline 2. Procedimiento: el paciente conoce procedimiento o propósito de la intervención & $86.7(78.1-95.3)$ & $100(0-0)$ & 0.01 \\
\hline 3. Registro de actividad: descripción del proceso & $38.3(26-50.6)$ & $93.3(87-94.6)$ & 0.001 \\
\hline 4. Registro de actividad: estado subjetivo del paciente & $63.3(51.1-75.5)$ & $90(82.4-97.6)$ & 0.001 \\
\hline 5. Registro de actividad: estado objetivo del paciente & $93.3(87-102.6)$ & $100(0-0)$ & 0.08 \\
\hline 6. Registro de actividad: plan de actuación & $65(52.9-77.1)$ & $96.7(92.2-101.2)$ & 0.001 \\
\hline 7. Registro de peso y talla del paciente & $65(52.9-77.1)$ & $91.7(84.7-98.7)$ & 0.001 \\
\hline 8. Valoración nutricional MNA: paciente sin riesgo nutricional & $60(47.6-72.4)$ & $83.3(73.9-92.7)$ & 0.01 \\
\hline
\end{tabular}

proceso asistencial, en el $36.7 \%$ no se describió el estado subjetivo, en el 35\% no se halló el plan de actuación, en el $35 \%$ no constaban el peso y talla, y el $40 \%$ de los pacientes no conocía a su médico responsable. Se encontraron diferencias entre los pacientes cuyo ingreso era de causa médica (no intervenidos) y los ingresados por causa quirúrgica; entre los pacientes con ingreso por motivo médico, el $70 \%$ no conocía al cirujano responsable de su caso, mientras que de aquellos con ingreso por motivo quirúrgico el $30 \%$ no lo identificaba $(p=0.034)$. Se observó riesgo de malnutrición no identificado en el $40 \%$ de los pacientes ingresados en el servicio de cirugía. En la figura 2 se muestran los incumplimientos ordenados según el diagrama de Pareto.

\section{Medida final}

Se observó una mejoría absoluta para todos los criterios salvo para "Conoce a su médico responsable» y «Descripción del estado objetivo del paciente», en los que la diferencia no llega a la significación estadística, pasando de 161 incumplimientos iniciales a 43 tras la implantación de las medidas de mejora. Existe una significativa mejoría (Fig. 3) en el registro de actividad, en la información recibida por los pacientes y en la detección de pacientes con problemas de malnutrición.

\section{Discusión}

En la actividad asistencial resulta imprescindible realizar una recogida de datos estructurada y estandarizada para obtener el mayor rendimiento en la identificación de posibles problemas activos y el establecimiento de planes de cuidados, así como evitar errores en el seguimiento y en la evaluación clínica de los pacientes. Esto es particularmente relevante en entornos donde el facultativo responsable de la atención a los pacientes cambia con relativa frecuencia (turnos de trabajo, asignación de tareas específicas, guardias, etc.), habiéndose documentado un déficit del adecuado registro de estos datos en distintos ámbitos ${ }^{5}$. En este sentido, se han ido desarrollando herramientas que permiten una mejor sistematización de la toma de datos en el entorno asistencial, destacando el desarrollo de dos conceptos en los que se ha basado este trabajo: la historia clínica orientada por problemas y la nota SOAP.

La historia clínica orientada por problemas fue descrita por Weed en $1964^{\circ}$. Sus principales componentes son el establecimiento de bases de datos, la elaboración de listas completas de problemas basada en esta base de datos y la generación de un plan para cada uno de los problemas identificados. Todo este flujo de información se centra en una nota de registro de la evolución clínica con formato SOAP.

La nota SOAP (Tabla 3) resulta un método muy efectivo de toma de datos ${ }^{11}$, que se centra en cuatros aspectos fundamentales en la atención al paciente hospitalizado. Este tipo de nota evolutiva o de valoración se utiliza en muchos ámbitos asistenciales sanitarios con el fin de garantizar la correcta documentación de la asistencia clínica, permitiendo el intercambio adecuado de información entre especialistas, así como la docencia y la evaluación de profesionales en formación y el análisis sistemático de los problemas asistenciales, al resultar un registro menos puramente narrativo que las notas «libres» habituales en la anamnesis o la evolución de los pacientes. Se ha difundido el uso integrado de la historia clínica orientada por problemas en la $\mathrm{HCE}$, en 


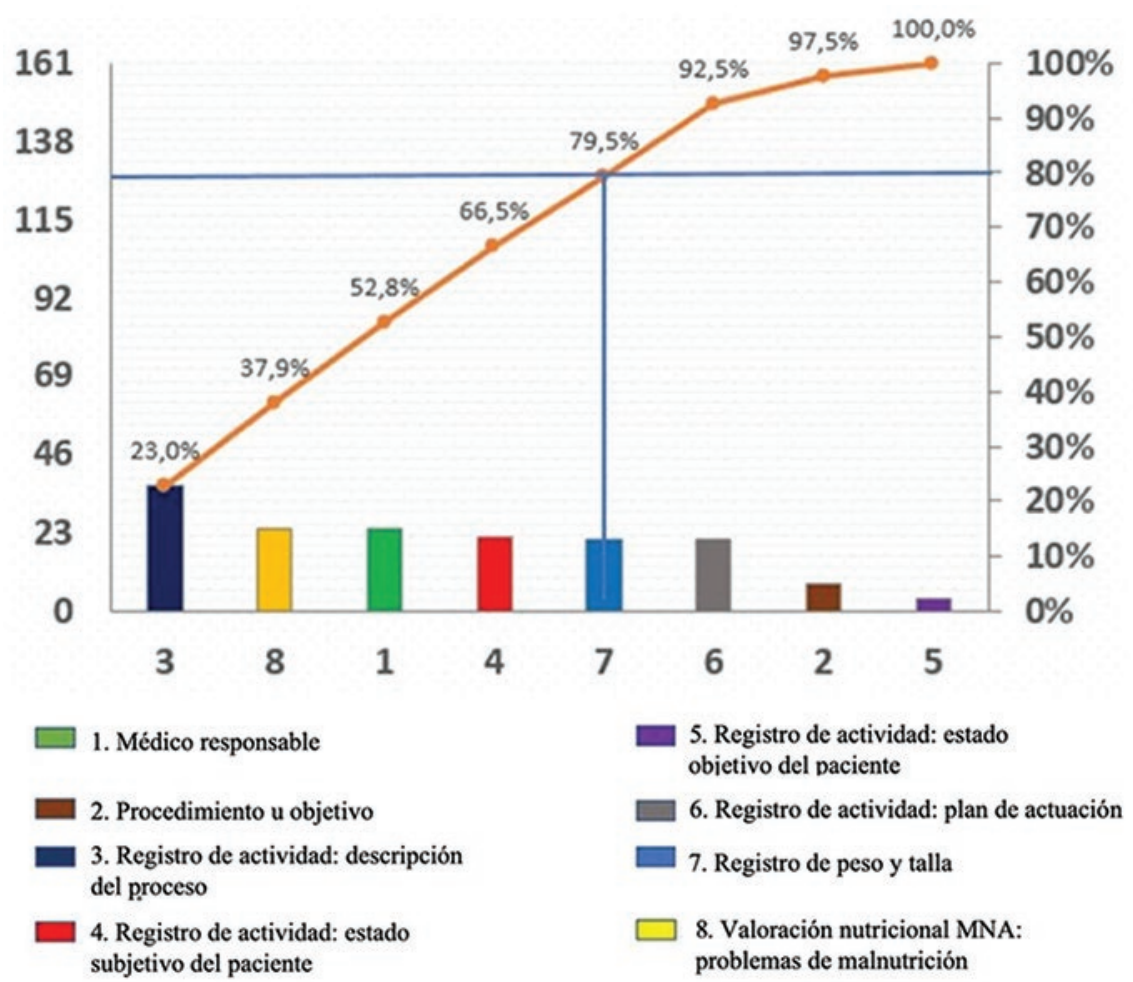

Figura 2. Diagrama de Pareto con las frecuencias de incumplimiento de criterios en la medida inicial.

particular las listas ordenadas de problemas, y el formato SOAP para las notas de evolución $n^{11-13}$.

El proceso de toma de decisiones en el ámbito asistencial quirúrgico en una sala de hospitalización resulta complejo, por estar sometido a constantes cambios, interacciones dinámicas y una notable ambigüedad. Siempre parte de los datos clínicos obtenidos del paciente, los cuales precisan una sistemática para su adquisición y registro que permita un adecuado intercambio de información entre profesionales². Estos registros sistematizados, como el que se introduce en nuestro estudio, también se consideran muy útiles para la educación médica ${ }^{14-16}$, ya que permiten a los estudiantes y residentes formarse y entrenarse para afrontar el proceso asistencial, una situación siempre ligada a unos altos índices de variabilidad y, por tanto, de difícil transmisión docente ${ }^{17}$, con buenos resultados publicados en diversos estudios ${ }^{3,18}$. Asimismo, el uso integrado de estos conceptos en la HCE ha permitido mejorar la calidad asistencial y la eficiencia, e incluso se propone como mecanismo para reducir los errores médicos ligándolos a estrategias en relación con la seguridad del paciente ${ }^{19} \mathrm{y}$ el gasto sanitario. No menos importante resulta el registro adecuado de datos clínicos (procedimientos, complicaciones, comorbilidad, etc.) para la adecuada codificación de las altas médicas ${ }^{6}$ y para la correcta cumplimentación de otro tipo de documentación clínica ${ }^{7}$. La mejora significativa en el registro de datos obtenidos en el pase de visita médica en la planta mediante el diseño y la implementación de una hoja formalizada ad hoc de recogida (proforma) se ha publicado por otros autores ${ }^{20}$. En nuestro estudio se aprecia una mejora sustancial en todos los parámetros estudiados respecto al registro de datos, en especial en la evaluación clínica (descripción del proceso del paciente) y en los aspectos relatados por el paciente (dimensión "subjetiva» que incluye los síntomas presentes, tales como dolor, náuseas, movilidad, etc.), lo que se entiende que puede incrementar la puesta en marcha de medidas asistenciales 0 terapéuticas correctamente orientadas por las necesidades del paciente y la expresividad clínica de su enfermedad.

Por otro lado, el correcto proceso de relación médico-paciente y de asignación del facultativo responsable durante la práctica médica supone un componente esencial de unos cuidados médicos completos, y constituye un requisito que debe ser cumplido en toda interacción médico-paciente, aunque esto resulta muy exigente en su efectiva realización en la práctica clínica ${ }^{21}$. En la atención en la planta de hospitalización quirúrgica debe respetarse y continuarse esta adecuada relación médico-paciente (que se inicia desde el 


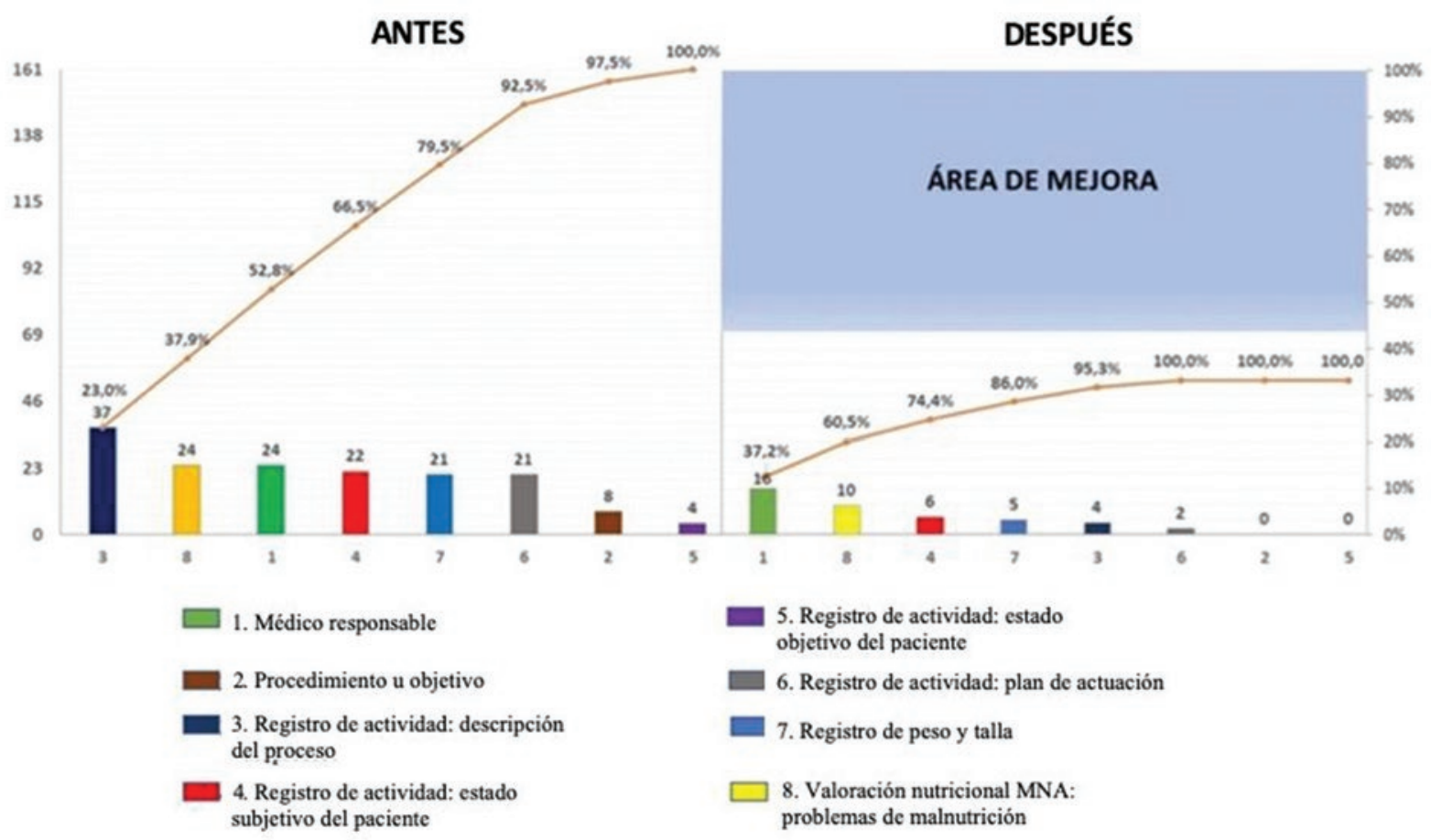

Figura 3. Diagrama de Pareto antes-después.

Tabla 3. Contenido de la nota SOAP

S Subjetivo: datos subjetivos obtenidos del paciente y otras personas cercanas a él (sintomatología, dolencias, grado de confort, etc.)

O Objetivo: datos objetivos obtenidos mediante observación, exploración física (incluidas las constantes vitales), estudios de diagnóstico, etc.

A Análisis: resumen del paciente y evaluación de su estado a través del análisis del problema, la posible interacción de los problemas y los cambios en el estado de los problemas

P Plan: hacer explícito el plan de atención que se llevará a cabo para el proceso diagnóstico-terapéutico del paciente y en relación con los problemas previamente identificados

ingreso) para conseguir una buena calidad asistencial, informando a los pacientes y haciéndoles partícipes de su proceso asistencial, lo que no solo aumenta la satisfacción de la asistencia clínica recibida, sino que también resulta fundamental para conseguir una atención humanizada y segura. En nuestro estudio, el porcentaje de pacientes que declaran desconocer al cirujano responsable de su atención (un $40 \%$ antes de la medida de mejora) o el procedimiento al que son 0 van a ser sometidos (un 20\%) puede parecer injustificadamente alto, pero otros estudios también ofrecen cifras similares ${ }^{21}$. La medida de mejora diseñada en nuestro estudio produjo una considerable mejora en estos parámetros, en particular en cuanto al conocimiento del procedimiento quirúrgico.
Finalmente, como tercera dimensión evaluada y atendida en nuestro ciclo de mejora, es conocido que, respecto a los pacientes ingresados en un hospital, entre un 30 y un $50 \%$ presentan malnutrición o probabilidad de desarrollarla, y que esta se asocia con un riesgo incrementado de complicaciones tras la cirugía, incluyendo un aumento en los días de hospitalización y recuperación ${ }^{22}$. Por este motivo, se considera que la identificación del riesgo nutricional es de extrema importancia, y puede hacerse mediante distintas herramientas y algoritmos de decisión ${ }^{23,24}$. Con este objetivo, para la valoración nutricional se empleó el MNA debido a su sencillez y efectividad, ya que permite estimar de manera simple, fácil y rápida la situación nutricional de los pacientes ${ }^{25}$, en especial de los pacientes geriátricos ${ }^{9,26}$, lo que tiene un valor probado en cuanto a pronóstico de morbimortalidad perioperatoria. La puesta en marcha de la medida de mejora a través de la entrevista personal con el paciente por parte del personal de enfermería de la planta quirúrgica consiguió establecer una rutina para la evaluación sistemática de este riesgo, lo que redundó en una disminución del $50 \%$ en el porcentaje de pacientes con riesgo nutricional identificados en nuestro estudio transversal antes-después de la implantación. Del mismo modo, la medida consiguió una mejora significativa en el registro de parámetros básicos en este contexto, tales como el peso 
y la talla del paciente, lo que no solo tiene una utilidad en cuanto a los aspectos nutricionales, sino también para el ajuste de la dosis de medicación, la fluidoterapia, etc.

Como limitaciones del estudio destaca que no se estableció un «equipo de mejora» estándar, sino que se promovió por parte de los autores del estudio, por lo que el grupo de profesionales pudo no implicarse lo suficiente. Además, no se profundizó en la implantación de una historia orientada a la identificación de problemas clínicos más allá del SOAP, ni se pudo integrar adecuadamente con el sistema de registro formal (formularios electrónicos) en la programación de la HCE vigente en nuestro hospital (Selene ${ }^{\circledR}$, Siemens), cuya adaptación, como han publicado otros autores ${ }^{4}$, no siempre resulta flexible ni libre de costes. Además, el efecto de las medidas puede estar sesgado por un efecto Hawthorne, y por último, idealmente, debe ser objeto de un programa de monitorización (programa de mejora continua de calidad).

\section{Conclusión}

En nuestro estudio destacan la identificación y la categorización por su importancia de diversas oportunidades de mejora en la asistencia a los pacientes durante la hospitalización en un servicio de cirugía general, tales como la ausencia de una sistemática para la recogida de datos o la identificación de problemas (con unos altos índices de flexibilidad y aleatoriedad de registro de actividad en las medidas iniciales), las dificultades en el registro de los aspectos relevantes en la herramienta utilizada de HCE, la ausencia de un sistema objetivo de evaluación nutricional y algunas notables debilidades en el proceso de consentimiento informado, como la simple identificación del médico responsable de la atención. La puesta en marcha de un ciclo de mejora de la calidad a través de medidas simples, como la sesión formativa con los cirujanos, la identificación y la comunicación objetiva al ingreso y durante la estancia hospitalaria del paciente, la integración de un sistema nemotécnico de registro de la actividad del cirujano en la planta (nota SOAP), el registro del peso y la talla en todo paciente con ingreso en la planta de cirugía al ingreso y semanalmente, y por último un proyecto de evaluación nutricional mediante la aplicación del MNA semanalmente por enfermería, se han mostrado muy eficaces para la mejora de la calidad de distintas dimensiones asistenciales en este ámbito concreto.

\section{Financiamiento}

La presente investigación no ha recibido ayudas específicas provenientes de agencias del sector público, del sector comercial ni de entidades sin ánimo de lucro.

\section{Conflicto de intereses}

Los autores declaran no tener conflicto de intereses que puedan afectar al trabajo remitido.

\section{Agradecimientos}

Los autores agradecen a María del Carmen Hernández Ferrandis (supervisora de planta de cirugía) y al resto del personal de enfermería de hospitalización quirúrgica su contribución para que este trabajo pudiera realizarse, y a José Aguilar por su gran dedicación y entusiasmo para llevar a cabo el estudio, porque sin él no hubiese sido posible.

\section{Responsabilidades éticas}

Protección de personas y animales. Los autores declaran que para esta investigación no se han realizado experimentos en seres humanos ni en animales.

Confidencialidad de los datos. Los autores declaran que han seguido los protocolos de su centro de trabajo sobre la publicación de datos de pacientes.

Derecho a la privacidad y consentimiento informado. Los autores han obtenido el consentimiento informado de los pacientes y/o sujetos referidos en el artículo. Este documento obra en poder del autor de correspondencia.

\section{Bibliografía}

1. Revilla F. Dimensiones de la calidad en sanidad. En: Cabo J, editor. Gestión de la calidad en las organizaciones sanitarias. Madrid: Fundación Mapfre; 2014. p. 1083-110.

2. Crebbin W, Beasley SW, Watters DAK. Clinical decision making: how surgeons do it. ANZ J Surg. 2013:83:422-8.

3. Dolan R, Broadbent P. A quality improvement project using a problem based post take ward round proforma based on the SOAP acronym to improve documentation in acute surgical receiving. Ann Med Surg. 2016; $5: 45-8$

4. Vigal Brey G, Trelles Martín A. Dos años de historia clínica electrónica. Experiencia en un hospital comarcal. Cir Esp. 2012;90:490-4.

5. Fernando KJ, Siriwardena AK. Standards of documentation of the surgeon-patient consultation in current surgical practice. Br J Surg. 2001; 88:309-12.

6. Gómez-Rosado J-C, Sánchez-Ramírez M, Valdés-Hernández J, Capitán-Morales LC, Del-Nozal-Nalda MI, Oliva-Mompean F. Importancia de la calidad del informe de alta en la gestión de una unidad clínica quirúrgica Cir Esp. 2013:91:378-83.

7. Prieto De Paula JM, Sanmamed Salgado N, Otero Rodríguez JA, Franco Hidalgo S, Romo Gil JM. Sobre la importancia del informe clínico en la gestión de una unidad clínica quirúrgica Cir Esp. 2013;91:617-8. 
8. Wright A, Sittig DF, McGowan J, Ash JS, Weed LL. Bringing science to medicine: an interview with Larry Weed, inventor of the problem-oriented medical record. J Am Med Inf Assoc. 2014;21:964-8.

9. Guigoz Y, Jensen G, Thomas D, Vellas B. The Mini Nutritional Assessment (MNA) review of the literature - what does it tell us? J Nutr Heal Aging. 2006;10:466-87.

10. Saturno PJ. Diseño de estudios del nivel de calidad y la investigación de causas. Componentes de una evaluación. Manual del Máster en Gestión de la Calidad en los Servicios de Salud. Ed. Universidad de Murcia; Murcia, España, 2008.

11. Pearce PF, Ferguson LA, George GS, Langford CA. The essential in an SOAP EHR age. Nurse Pract. 2016;41:29-36.

12. Cillessen FHJM, de Vries Robbé PF. Modeling problem-oriented clinical notes. Meth Inf Med. 2012;51:507-15.

13. Shoolin J, Ozeran L, Hamann C, Bria W. Association of Medical Directors of Information Systems consensus on inpatient electronic health record documentation. Appl Clin Inform. 2013;4:293-303.

14. Davies M, Schonder K, Meyer S, Hall D. Changes in student performance and confidence with a standardized patient and standardized colleague interprofessional activity. Am J Pharm Educ. 2015;79:1-7.

15. Mitsuishi F, Young JQ, Leary M, Dilley J, Mangurian C. The systems SOAP note: a systems learning tool. Acad Psychiatry. 2016:40:164-71.

16. Reznich CB, Wagner DP, Noel MM. A repurposed tool: the programme evaluation SOAP note. Med Educ. 2010;44:298-305

17. Apramian $\mathrm{T}$, Cristancho $\mathrm{S}$, Watling $\mathrm{C}$, Ott M, Lingard L. "They have to adapt to learn": surgeons' perspectives on the role of procedural variation in surgical education. J Surg Educ. 2015;73:339-47.
18. Jeanne E, Frenzel P. Using electronic medical records to teach patient-centered care. Am J Pharm Educ. 2010;74:1-6.

19. Weiss PM, Lara-Torre E, Murchison AB, Spotswood L. Expanding the SOAP note to SOAPS (with $S$ for safety): a new era in real-time safety education. J Grad Med Educ. 2009;1:316-8.

20. Thompson AG, Jacob K, Fulton J, McGavin CR. Do post-take ward round proformas improve communication and influence quality of patient care? Postgrad Med J. 2004;80:675-6.

21. Ochieng J, Buwembo W, Munabi I, Ibingira C, Kiryowa H, Nzarubara G, et al. Informed consent in clinical practice: patients' experiences and perspectives following surgery. BMC Res Notes. BioMed Central; 2015;8:1-6.

22. Izaola O, De Luis Román DA, Cabezas G, Rojo S, Cuéllar L, Terroba MC, et al. Mini Nutritional Assessment (MNA) como método de evaluación nutricional en pacientes hospitalizados. An Med Interna. 2005;22:313-6.

23. de Kruif JTCM, Vos A. An algorithm for the clinical assessment of nutritional status in hospitalized patients. Br J Nutr. 2003;90:829-36.

24. Ravasco $\mathrm{P}$, Anderson $\mathrm{H}$, Mardones $\mathrm{F}$. Métodos de valoración del estado nutricional. Nutr Hosp. 2010;25(Supl 3):57-66.

25. Villamayor Blanco L, Llimera Rausell G, Jorge Vidal V, González Pérez-Crespo C, Iniesta Navalón C, Mira Sirvent MC, et al. Valoración nutricional al ingreso hospitalario: iniciación al estudio entre distintas metodologías. Nutr Hosp. 2006;21:163-72.

26. Kaiser M, Bauer J, Ramsch C, Uter W, Guigoz Y, Cederholm T, et al. Validation of the Mini Nutritional Assessment short-form (MNA-SF): a practical tool for identification of nutritional status. J Nutr Heal Aging. 